\title{
Multifaceted Analysis of Fine-Tuning in a Deep Model for Visual Recognition
}

\author{
XIANGYANG LI, CAS and University of Chinese Academy of Sciences \\ LUIS HERRANZ, Universitat Autònoma de Barcelona \\ SHUQIANG JIANG, CAS and University of Chinese Academy of Sciences
}

In recent years, convolutional neural networks (CNNs) have achieved impressive performance for various
visual recognition scenarios. CNNs trained on large labeled datasets not only obtain significant performance
on most challenging benchmarks but also provide powerful representations, which can be used for a wide
range of other tasks. However, the requirement of massive amounts of data to train deep neural networks
is a major drawback of these models, as the data available are usually limited or imbalanced. Fine-tuning
is an effective way to transfer knowledge learned in a source dataset to a target task. In this article, we
introduce and systematically investigate several factors that influence the performance of fine-tuning for
visual recognition. These factors include parameters for the retraining procedure (e.g., the initial learning
rate of fine-tuning), the distribution of the source and target data (e.g., the number of categories in the source
dataset, the distance between the source and target datasets), and so on. We quantitatively and qualitatively
analyze these factors, evaluate their influence, and present many empirical observations. The results reveal
insights into what fine-tuning changes CNN parameters and provide useful and evidence-backed intuition
about how to implement fine-tuning for computer vision tasks.

CCS Concepts: • Computing methodologies $\rightarrow$ Computer vision; Image representations; Object recognition; Neural networks;

Additional Key Words and Phrases: Deep learning, convolutional neural network, image classification, finetuning

\section{ACM Reference format:}

Xiangyang Li, Luis Herranz, and Shuqiang Jiang. 2020. Multifaceted Analysis of Fine-Tuning in a Deep Model for Visual Recognition. ACM/IMS Trans. Data Sci. 1, 1, Article 4 (January 2020), 22 pages.

https://doi.org/10.1145/3319500

\footnotetext{
This work was supported in part by the National Natural Science Foundation of China under grant 61532018, in part by the Beijing Natural Science Foundation under grant L182054, and in part by the National Program for Special Support of Eminent Professionals and the National Program for Support of Top-notch Young Professionals.

Authors' addresses: X. Li and S. Jiang, Key Laboratory of Intelligent Information Processing of Chinese Academy of Sciences (CAS), Institute of Computing Technology, CAS, Beijing 100190, China, and University of Chinese Academy of Sciences, Beijing 100049, China; emails: xiangyang.li@vipl.ict.ac.cn, sqjiang@ict.ac.cn; L. Herranz, Learning and Machine Perception (LAMP) Group of the Computer Vision Centre, Universitat Autònoma de Barcelona, 08193 Bellaterra, Barcelona, Spain; email: lherranz@cvc.uab.es.

Permission to make digital or hard copies of all or part of this work for personal or classroom use is granted without fee provided that copies are not made or distributed for profit or commercial advantage and that copies bear this notice and the full citation on the first page. Copyrights for components of this work owned by others than ACM must be honored. Abstracting with credit is permitted. To copy otherwise, or republish, to post on servers or to redistribute to lists, requires prior specific permission and/or a fee. Request permissions from permissions@acm.org. (C) 2020 Association for Computing Machinery.

2577-3224/2020/01-ART4 \$15.00

https://doi.org/10.1145/3319500
} 


\section{INTRODUCTION}

Visual recognition is a fundamental concern of computer vision in the big data age. Over the past years, it has achieved significant progress due to the rapid development of ubiquitous sensing technologies of collecting image data and the availability of large computational resources to train big models. One of the huge successes is deep convolutional neural networks (CNNs), which have achieved excellent performance on a large number of visual tasks, such as recognition [17-19, 22-24, 26, 28, 46-48], object detection [11, 12, 41, 43], and segmentation [21, 31]. These models are mainly built upon the convolution operation, which extracts discriminative and informative features by gradually integrating spatial and channel information within local receptive fields. In this manner, they learn visual representations layer by layer, where low features (e.g., color blobs, edges, and simple shapes, which are applicable to many datasets and tasks) are general and high-layer features are specific, which depend much on the target dataset and task [58].

Krizhevsky et al. [26] first trained AlexNet [26] on ImageNet for the large-scale visual recognition challenge in 2012 (ILVRC 2012) [42]. Their successes are that they not only obtain significant performance on most challenging datasets $[42,61]$ but also provide powerful representations that can be used for other tasks or different datasets [7, 9, 14, 44, 57]. Since then, CNNs have been successfully applied to numerous visual tasks. The approaches employing CNNs can be divided into two categories according to whether they have abundant data to train deep neural networks.

With the availability of large annotated target datasets such as ImageNet [42] and Places [61], the first way is to directly train a model from scratch on the target dataset. After the success of AlexNet [26], many complex architectures have been proposed, such as VGGNet [46], GoogLeNet [48], ResNet [18], and SENet [22]. These high-capacity models have brought excellent progress by mainly increasing network depth and width, as well as enhancing the connection between different layers and channels of feature maps. For example, Symonyan and Zisserman [46] investigate the effect of network depth with the recognition accuracy and propose a 19-layer architecture that outperforms previous neural networks with a huge margin. Hu et al. [22] focus on the channel interdependence in feature maps and propose SENet [22],which obtained the best performance in ILSVRC 2017. Luan et al. [33] propose Gabor convolutional networks (GCNs), which incorporate Gabor filters to CNNs to enhance deep feature representations with steerable orientation and scale capacities. Wu et al. [55] propose a model to learn unified hash codes and deep hash functions. Liu et al. [29] design a multiscale architecture based on the network simulation of wavelet multiresolution analysis. Wu et al. [56] introduce a deep model that integrates deep convolutional networks with binary latent representation learning. Li et al. [27] combine the $\mathrm{CNN}$ and recursive neural network (RNN) for visual recognition. Wu et al. [54] propose a model where feature learning and hash function learning are jointly integrated.

However, for many object recognition or scene classification datasets, there are not so many available labeled images to feed such big models. For these situations, the second way is to just employ a pretrained CNN as a feature extractor. Approaches in this category first take the whole image as the input for a pretrained $\mathrm{CNN}$ to extract high-layer activations as image representations and then utilize them to train simple classifiers to obtain recognition results. The combination of deep representations and a simple classifier has been a preferred solution for many visual recognition tasks [7, 9, 14, 30, 44, 57]. For example, Donahue et al. [9] suggest that the deep CNN trained on ImageNet is an effective feature extractor and provides large evidence to support this claim. Gong et al. [14] utilize a CNN to extract local patch features at multiple scales and then pool them to image-level features. Their method harvests deep activation features and obtains significant performance on many datasets. Liu et al. [30] also reveal that the activations of convolutional layers are useful image representations. 


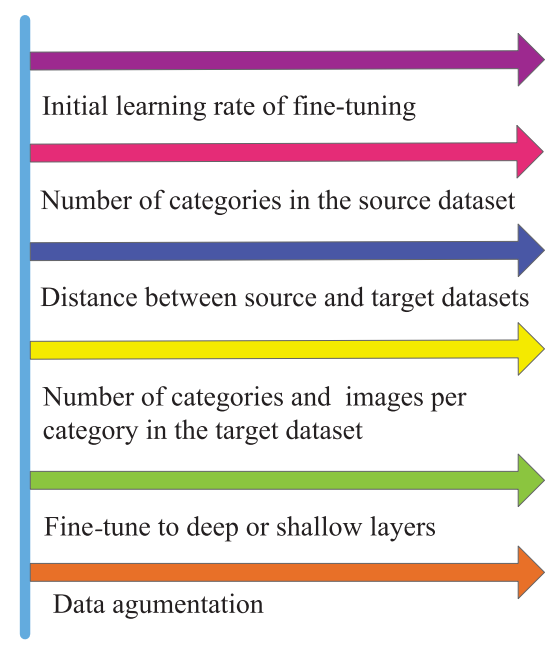

Fig. 1. Factors that influence the performance of fine-tuning.

Although directly using activations of a pretrained model has good performance for many tasks, fine-tuning a pretrained model on one target dataset can further improve performance by making the features more specific to the target task [1, 7, 12, 15, 16, 36, 52, 62]. Girshick et al. [12] remove the specific 1,000-category classification layer of a CNN that is trained on ImageNet and replace it with a randomly initialed $(\mathrm{N}+1)$-category classification layer (where $\mathrm{N}$ is the number of categories in the target dataset and the extra one category is for the background). To learn the new parameters, they retrain the modified model on the extracted region proposal dataset, with unchanged parameters initialized from the pretrained one. Their work indicates that fine-tuning is fruitful. Chatfield et al. [7] present rigorous comparisons of the handcrafted features, CNN-based features, and CNN-based features after fine-tuning on many datasets. Their work indicates that the fine-tuned CNN-based features outperform the others by a large margin. The work of Agrawal et al. [1] and Gupta et al. [15] also demonstrates that fine-tuning a pretrained CNN can significantly improve performance.

To employ the inherent knowledge of a CNN that trained on a big source dataset, it is intuitive to apply fine-tuning because it is a reasonable and effective way to make full use of a pretrained model and alleviate the scarce of training data. In this article, we explore factors that influence the performance of fine-tuning, as illustrated in Figure 1. There are many factors that influence the performance of fine-tuning, which include parameters for the retraining procedure (e.g., the initial learning rate (LR) of fine-tuning), the distribution of the source and target data (e.g., the number of categories in the source dataset, the distance between the source and target datasets), and so on. To address the problem of how to fine-tune properly to obtain satisfactory performance on different classification tasks with various target datasets, in this work we quantitatively and qualitatively analyze these factors respectively. We artificially select different source and target datasets with different constraints and then conduct fine-tuning on these datasets. To the best of our knowledge, this is the first systematic investigation of these factors. The main empirical observations include the following aspects.

First, with roughly the same distance to the target dataset, the bigger the number of categories in the source dataset, the better performance fine-tuning obtains. The pretrained procedure works as a regularization to make a better generalization from the source dataset. For a fixed target dataset, the more categories the source dataset has, the more knowledge is learned. More categories make the pretrained model have better generalization ability, bringing better performance of fine-tuning. 
Second, when the source dataset is fixed, the performance of fine-tuning increases with more training examples exposed to the retraining of the pretrained model. In addition, the gain in finetuning versus training the network from scratch decreases with the increase of retraining examples. With more training examples, the parameters in different models are adjusted to fit the target data, so the difference decreases.

Last, we manually select source datasets that contain the same number of categories but have different similarity to the target dataset. The results show that the more similarity between the source and target datasets, the better performance fine-tuning obtains. We analyze the characteristic of different models at both the filter level and layer level, and we show their sensitivities to the target dataset. We also qualitatively show the differences among these different models.

In the following, we give an overview of related work in Section 2. Sections 3 and 4 present our experimental settings and results. We offer our conclusion in Section 5.

\section{RELATED WORK}

Our work is related to the work of exploring the transferability of pretrained deep models. In this section, we briefly review the related work about unsupervised pretraining, domain adaptation, and transfer learning.

\subsection{Unsupervised Pretraining}

Greedy layerwise pretraining of unsupervised learning followed with global fine-tuning of supervised learning is an essential component for deep learning, as the pretraining procedure can introduce useful prior knowledge. The training strategies for deep models like deep belief networks (DBNs) [20], stacked auto-encoders (SAEs) [6], and stacked denoising auto-encoders (SDAEs) [51] are both based on a similar approach. First, each layer learns a transformation of its input independently. Second, all parameters are fine-tuned with respect to a training criterion. Compared with randomly initialized approaches, the gain obtained through pretraining is impressive. The effect of unsupervised pretraining can be explained as regularization, and it guides the learning toward basins of attraction of minima that support better generalization from the training examples [10]. Whereas in the conventional training procedure of CNNs, there is no unsupervised pretraining stage. However, when fine-tuning a pretrained $\mathrm{CNN}$ model on a target dataset, we can regard the supervised pretraining as a substitution of the unsupervised pretraining. In this work, we empirically show the influence of various factors when conducting fine-tuning.

\subsection{Domain Adaptation}

Domain adaption focuses on how to deal with data sampled from different distributions [4,38] (e.g., from product shot images to real-world photos), thus compensating for their mismatch. Since the theoretical analysis by Ben-David et al. [5], there has been a lot of research related to this area [8, 13, 50]. Glorot et al. [13] demonstrate that the features extracted from SDAE are beneficial for domain adaptation. Chopra et al. [8] propose a method that learns image representations with respect to a domain shift by generating a lot of intermediate datasets, which are obtained by interpolating between the source dataset and the target dataset. More similar to our work, Tzeng et al. [50] propose a new $\mathrm{CNN}$ with a new adaptation layer and an auxiliary domain confusion loss to learn domain-invariant representation. Long et al. [32] propose deep adaptation networks (DANs) to learn transferable features with statistical guarantees. Their proposed method can also scale linearly by unbiased estimate of kernel embedding. The aim of our work is to study the factors involved in fine-tuning but not to obtain the best performance. We focus on one fixed architecture (i.e., AlexNet) and conduct experiments on subdatasets that are manually selected from the 
Table 1. Architecture of the CNN Used in Our Experiments

\begin{tabular}{c|cccccccccccccccccccc}
\hline Layer & 1 & 2 & 3 & 4 & 5 & 6 & 7 & 8 & 9 & 10 & 11 & 12 & 13 & 14 & 15 & 16 & 17 & 18 & 19 & 20 \\
\hline Name & C1 & R1 & P1 & N1 & C2 & R2 & P2 & N2 & C3 & R3 & C4 & R4 & C5 & R5 & P5 & FC6 & R6 & FC7 & R7 & FC8 \\
Type & C & R & P & N & C & R & P & N & C & R & C & R & C & R & P & FC & R & FC & R & FC \\
Channels & 96 & 96 & 96 & 96 & 256 & 256 & 256 & 256 & 384 & 384 & 384 & 384 & 256 & 256 & 256 & 4,096 & 4,096 & 4,096 & 4,096 & 1,000 \\
Size & 11 & - & 3 & - & 5 & - & 3 & - & 3 & - & 3 & - & 3 & - & 3 & - & - & - & - & - \\
Parameters & 11,712 & - & - & - & 6,656 & - & - & - & 3,840 & - & 3,840 & - & 2,560 & - & - & $37,752,832$ & - & $16,781,312$ & - & $4,097,000$ \\
\hline Percentage & 0.01997 & & 0.01135 & & & 0.006546 & 0.006546 & 0.004364 & & 64.36 & & 26.61 & & 6.984 \\
\hline
\end{tabular}

Note: $\mathrm{C}$ indicates the convolutional layer. $\mathrm{R}$ indicates the nonlinear layer with the activation function of Relu. P indicates the max pooling layer. $\mathrm{N}$ indicates the normalization layer. FC indicates the fully connected layer.

ImageNet [42] dataset. Our experiments are dedicated to analyzing the factors that influence the performance of fine-tuning.

\subsection{Transfer Learning}

Transfer learning focuses on the possibility to utilize useful knowledge learned from a source task to master a target task [37]. Different from domain adaptation, an essential requirement for successful knowledge transfer is that the source domain and the target domain should be closely related $[38,39]$. Transfer learning has received much attention recently, and many approaches based on CNNs have been proposed in the computer vision community [2, 16, 35, 58, 59]. The knowledge transferred from CNNs can be categorized into two kinds. The first one is in the form of feature representations. These approaches use a pretrained $\mathrm{CNN}$ as a feature extractor and then directly utilize these extracted features for the target task $[7,9,44]$. The second one is in the form of model parameters. Oquab et al. [35] propose a framework that removes the output layer of the $\mathrm{CNN}$ and adds an additional module formed by two fully connected layers. Their work indicates that this procedure is an effective way to use the knowledge in a pretrained model. Yosinski et al. [58] study the transferability of CNNs in each layer. Wang et al. [53] reuse the parameters of a pretrained model and add new units to increase its depth and width. Most similar to our work, Azizpour et al. [2] investigate many factors affecting the transferability of CNNs, such as the width and depth of different architectures and the diversity and density of training data. Fine-tuning is just one investigated issue in their work. In this work, we systematically analyze the factors that affect the performance of fine-tuning based on one model with a fixed structure and shed light on the inner working mechanism of fine-tuning.

\section{EXPERIMENTAL SETUP}

The architecture of the CNN in our experiments is almost the same as the one proposed by Krizhevsky et al. [26]. It has five convolutional layers and three fully connected layers. Table 1 details the structure and parameters of it. In our experiments, we train and fine-tune the CNN with the Caffe open source framework [25].

For training the model on the source dataset, the learning choices are the same as those of Krizhevsky et al. [26]. The weights in each layer are initialized from a zero-mean Gaussian distribution with deviation of 0.01 , except the deviations of layers fc6 and fc7 are 0.005 . The neuron biases in conv2, conv4, and conv5, as well as all fully connected layers, are initialized with the constant 1 , whereas the biases in other layers are initialized with the constant 0 . The momentum is 0.9 , and the weight decay is 0.0005 . The LR at the beginning is 0.01 (for training models from scratch), and after every 20 cycles it is divided by the constant 10 . We train the CNN for roughly 100 cycles. To reduce overfitting, first there are two dropout layers with a dropout ratio of 


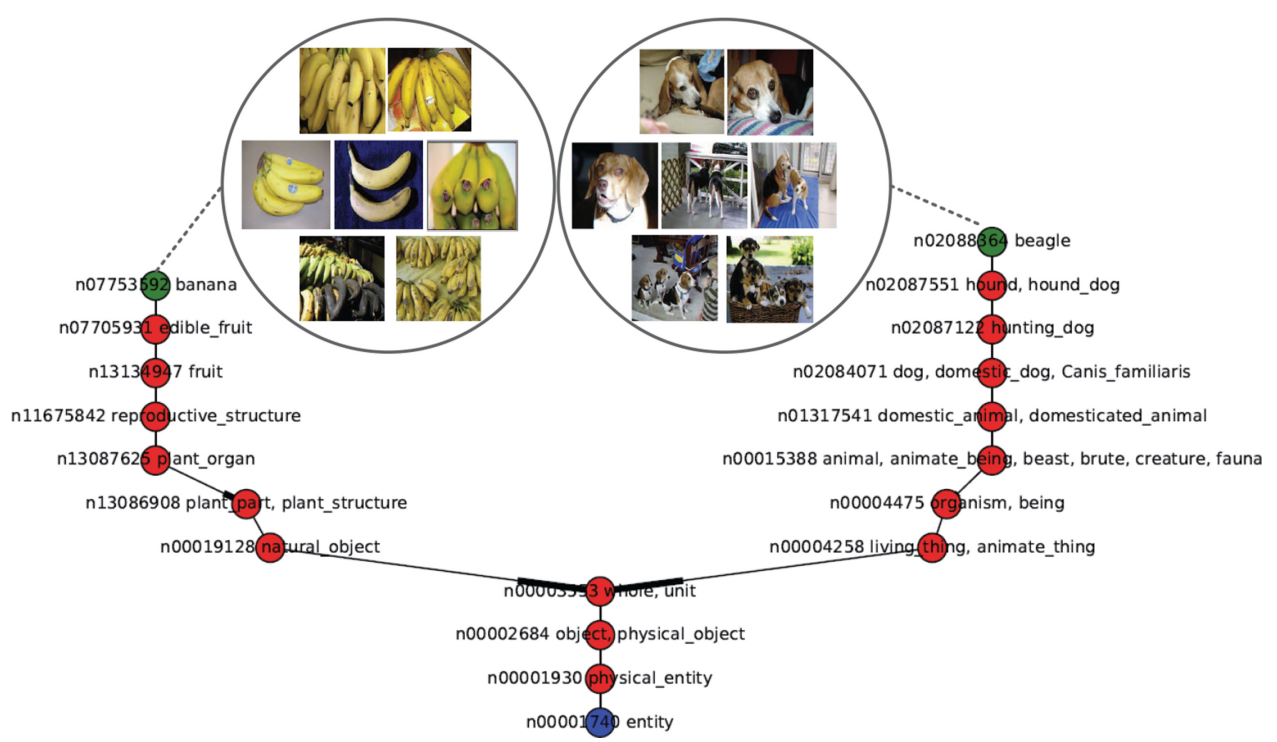

Fig. 2. The parent classes of the banana class and the beagle class. The green leaf nodes are the target classes, and the blue root node is the entity class that is the root of WordNet. The red nodes are the middle classes through which the leaf nodes can reach the root.

0.5 followed by the layers of fc6 and fc7. Second, we randomly crop $227 \times 227$ pixels from the $256 \times 256$ input image and randomly mirror it in each forward and backward processing.

For fine-tuning the pretrained model on the target dataset, we remove the last fully connected layer that is specific to the source task and replace it with a new randomly initialized one with $C$ units (where $C$ is the number of categories in the target task). After this, we use stochastic gradient descent (SGD) to continuously train (i.e., retrain) the modified model on the target dataset. In contrast to the work of Yosinski et al. [58], the parameters copied from the pretrained model are optimized with respect to the target task in our experiments, whereas these transferred parameters are fixed during retraining in their work.

We analyze the factors that affect fine-tuning mainly on the 1,000 ImageNet classes [42] dataset (ILSVRC2012), as shown in Figure 3. It has a total of 1.28 million images with abundant semantic diversity and density. To find subdatasets with different distances (e.g., A is a dataset that only contains domestic dogs, B is a dataset that contains many other animals such as cats and sheep, and $\mathrm{C}$ is a dataset that contains many traffic instruments), we define that the distance between $\mathrm{A}$ and $\mathrm{B}$ is smaller than the distance between $\mathrm{A}$ and $\mathrm{C}$ qualitatively, as objects in $\mathrm{A}$ and $\mathrm{B}$ both have eyes, legs, and tails, whereas objects in $\mathrm{C}$ do not have them. We draw out the tree structure of 1,000 ImageNet classes according to WordNet [34]. For every category in the 1,000 classes, it belongs to one synset in WordNet. We can find its parent classes recursively until reaching the node of entity that is the root of the WordNet. For example, the parent classes of the "n02088364 beagle" class and the class of "n07753592 banana" are showed in Figure 2. The tree structure of the entire 1,000 classes is illustrated in Figure 3. In the following, we select subdatasets from the 1,000 ImageNet classes with different constraints.

\section{FACTORS THAT AFFECT THE PERFORMANCE OF FINE-TUNING}

Many factors affect the performance of fine-tuning, such as the number of categories in the source dataset, the distance between the source and target datasets, the number of categories in the target 


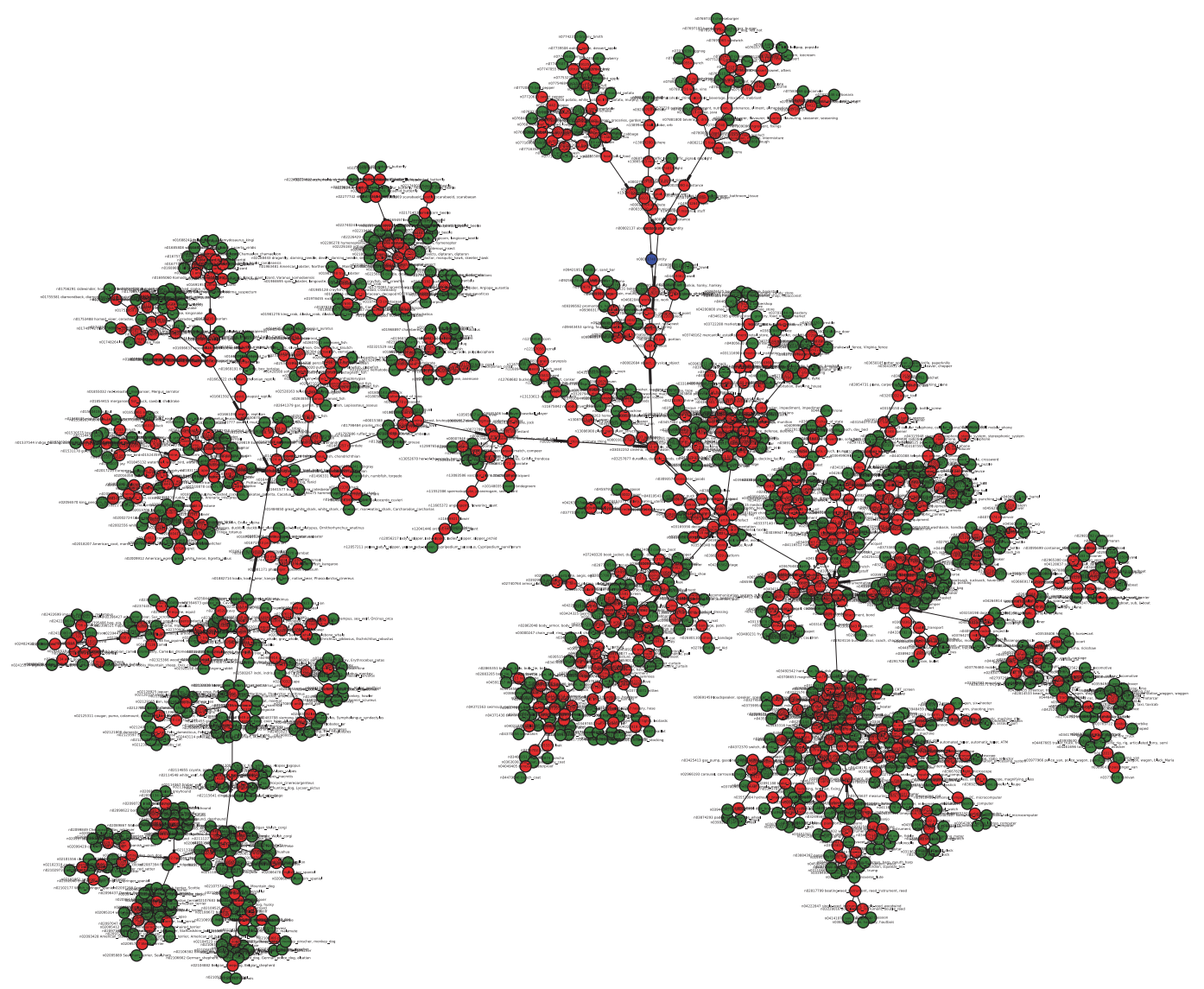

Fig. 3. The tree structure of 1,000 ImageNet classes. The green leaf nodes are the classes in the 1,000 ImageNet classes. The red nodes are the middle classes through which the leaf nodes can reach the root. The blue root node is the entity class that is the root of WordNet.

dataset, and the number of training examples per category in the target dataset. In our article, we individually analyze the effect of each factor. For training models from scratch and fine-tuning models from pretrained ones, we use all images (which belong to the selected categories) in the training set of ImageNet. We first train a set of models on different datasets and then fine-tune these model on the target datsets. For testing, we use 50 images per category, which are the same as the validation set of ImageNet.

\subsection{The Initial LR of Fine-tuning}

Fine-tuning is implemented by initializing a network with parameters optimized on one source dataset, except the last task-specific layer, which is randomly initialized. Then, using the target training samples, the parameters of the network are updated. When using SGD to optimiz the weights, the initial LR has a big influence on the learning procedure. As previous work has mentioned $[2,12]$, the initial LR at the beginning should be smaller than the initial LR used to optimize the weights on the source dataset. This strategy ensures that the prior knowledge learned in the source dataset is not clobbered. 

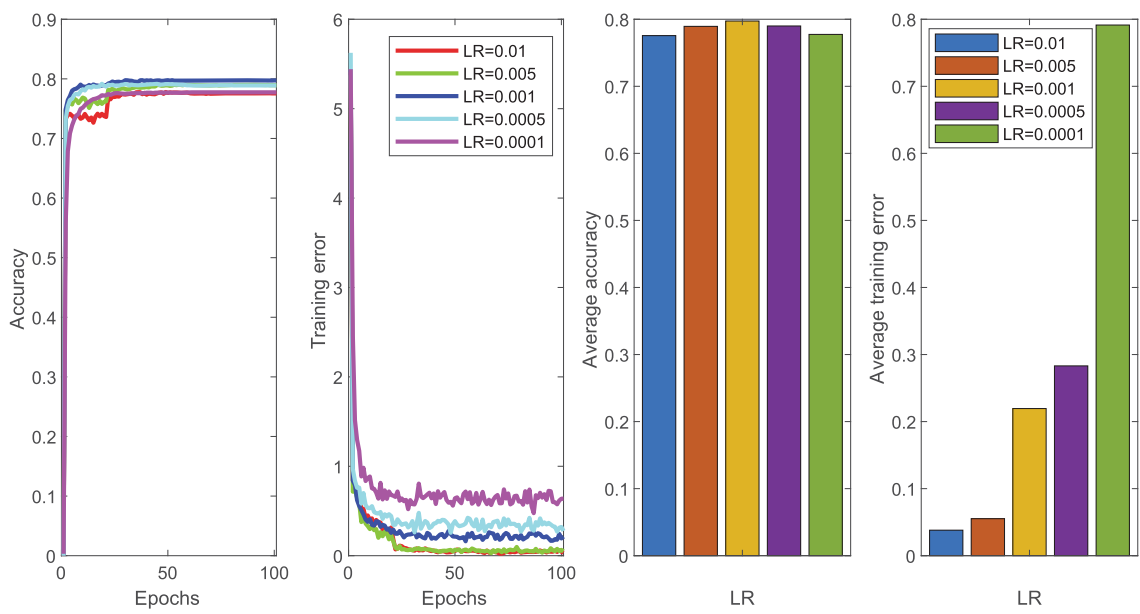

Fig. 4. The effect of the initial LR on the performance of fine-tuning. The first column is the performance on the test set when fine-tuning the pretrained model with different initial LRs. The second column is the training error (i.e., training loss). The third and fourth columns are the average accuracy and the average training error. The experiments are conducted independently five times.

However, to what extent does the initial LR affect the fine-tuning performance? In this section, we experimentally investigate this factor. We randomly select 100 classes from the 1,000 ImageNet classes as the target dataset. Then, we train the model from scratch on the remaining 900 categories. After this, we fine-tune the pretrained model on the 100-class target dataset with decreasing initial LRs: $0.01,0.005,0.001,0.0005$, and 0.0001 . The experiments are conducted independently five times (i.e., five target datasets and five source datasets). The results are shown in Figure 4 . When the initial LR is set to 0.02 or bigger, the learning procedure does not converge as we have tried in our experiments. Thus, we set 0.01 as the biggest initial LR. The remarkable observations are as follows. First, bigger initial LRs bring smaller training errors. Second, starting with a big initial LR, there are some bumps in the early training epochs. This indicates that the basins of attraction of minima are reconstructed. Although the training error is much smaller, the generalization ability is worse, as the test accuracy is not good enough compared with a moderate initial LR. Third, when starting with a very small LR, the training error is much bigger and the test accuracy is smaller. This indicates that the learning procedure is not plenitudinous. When starting with the initial LR of 0.001 , the learning procedure is mild, and it obtains the best performance. Therefore, in the rest of our experiments, we fixed the initial LR with 0.001 .

\subsection{Number of Categories in the Source Dataset}

Previous work has suggested that increasing the training data can significantly improve performance $[2,35]$. In this section, we investigate the effect of the number of categories in the source dataset for fine-tuning. First, we randomly select 100 categories from ImageNet as the fixed target dataset. Then, we randomly select $800,500,200,100$, and 80 categories, respectively, from the remaining categories as different source datasets. Thus, the distances between these source datasets and the target dataset are roughly the same.

After obtaining different kinds of pretrained models that are trained on these selected source datasets, we fine-tune these models on the fixed target dataset. Figure 5 is a plot of the accuracy and training error when fine-tuning different pretrained models on a fixed target dataset. We also 

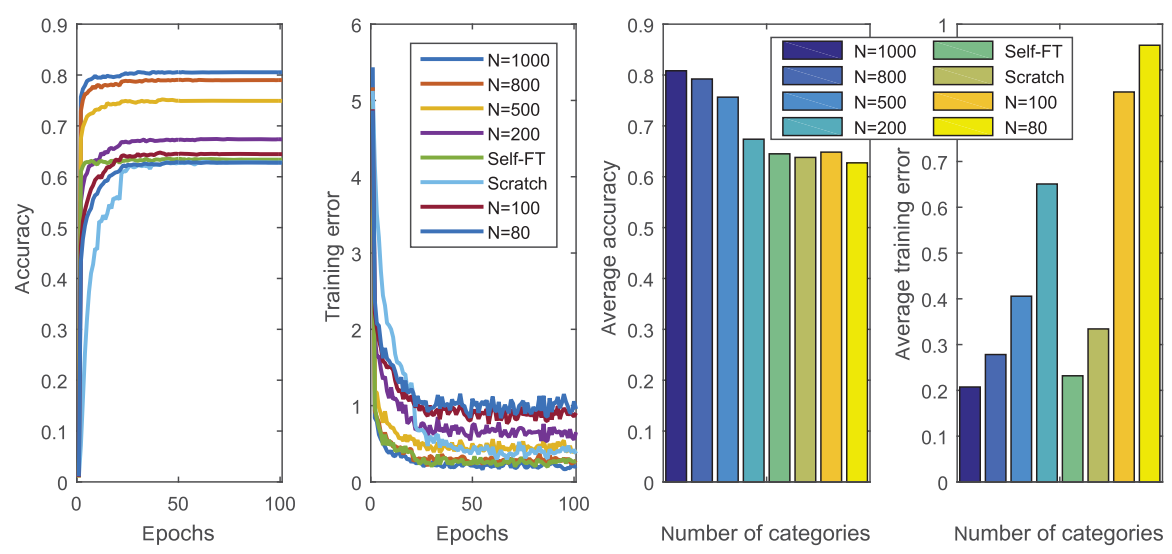

Fig. 5. The effect of the number of categories in the source dataset when fine-tuning from different pretrained models on the fixed target dataset. The first column is the accuracy, and the second column is the training error (i.e., training loss). The third and fourth columns are the average values. We run the experiments with the same settings five times.

Table 2. Performance (Mean and Variance) of Fine-Tuning Different Pretrained Models on the Fixed Target Dataset

\begin{tabular}{c|cccc}
\hline Number & 1,000 & 800 & 500 & 200 \\
\hline Accuracy (\%) & $80.94 \pm 0.75$ & $79.25 \pm 0.23$ & $75.97 \pm 1.17$ & $66.76 \pm 1.71$ \\
\hline \hline Number & SF-FT & Scratch & 100 & 80 \\
\hline Accuracy (\%) & $63.53 \pm 2.33$ & $62.47 \pm 2.50$ & $63.67 \pm 1.67$ & $61.35 \pm 2.02$ \\
\hline
\end{tabular}

Note: We conduct the experiments respectively five times.

conduct the experiments independently five times, and Table 2 presents the average performance. Several observations can can be drawn from these results.

First, when we fine-tune a pretrained model on a dataset, it dramatically converges faster than training it from scratch, especially when the source dataset has many categories. The explanation is that the model pretrained on dataset that contains enough categories has a better basin of attraction of minima, and its parameters can efficiently be adapted to new tasks with few changes.

Second, a network trained on more categories contains more knowledge, and the generalization ability of the network is much better. As we can seen in Figure 5, with the decrease of the number of categories in the pretrained model, the performance of fine-tuning decreases and the training error becomes larger. For example, the model trained on 1,000 categories $(N=1,000)$ has the best performance, and the training error is the smallest. When the pretrained number of categories is $500(N=500)$, the training error is larger and the performance decreases. But it is obvious that even though the training error $(N=100)$ is larger, the performance is still better than the performance of training the network from scratch. When fine-tuning from itself (continued training on the target dataset itself), the training error becomes smaller, but the test accuracy is almost the same with the accuracy of training the network from scratch.

Last, when fine-tuning from a deficient model that trained on a source dataset that only has a small number of categories $(N=80)$, the performance is even worse than training the model from scratch. This can be attributed to the perishing generalization ability of the pretrained model and the small LR of fine-tuning. 


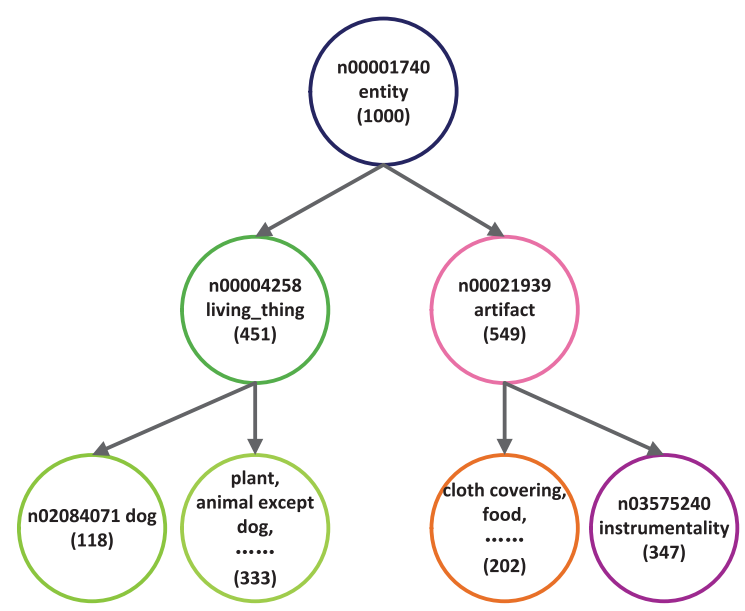

Fig. 6. Different branches of the 1,000 categories in ImageNet. The integer in the parentheses indicates the number of the categories the branch contains. The number in the beginning of the node is the corresponding WordNet ID.

With roughly the same distance to the target dataset, the bigger the number of categories in the source dataset, the better performance fine-tuning obtains, as shown in the results. In other words, the procedure of pretraining a model works as a regularization, making better generalization from the source dataset. The more knowledge learned in the source dataset, the better generalization ability the pretrained model has.

\subsection{Distance Between the Source and Target Datasets}

It is well known that dataset bias is a very common issue in visual recognition $[2,49,50,58]$. However, when fine-tuning pretrained models on a target dataset, what is the relationship of the corresponding performance and the distance between the source and target datasets? It is meritorious to expose what happens when fine-tuning from different pretrained models. In this section, we investigate this factor qualitatively and quantitatively and reveal insights into what happens when conducting fine-tuning.

Measuring the distance between two datasets is crucial in image recognition. We consider the way used by Yosinski et al. [58]. More precisely, we manually select nodes in different branches in the tree structure of the 1,000 categories in ImageNet, thus forming subdatasets that have a different distance to a fixed target dataset, as illustrated in Figure 6. For example, the 1,000 categories are divided into two branches: one is living things, which contains 451 categories, and the other one is artifacts, which contains 549 categories. We first select two fixed datasets with 100 categories and then select two datasets with different distances with 500 categories for each of the target datasets. The procedures to obtain the datasets are as follows. First, the dog branch has 118 categories (e.g., rhodesian ridgeback, papillon, and saint bernard), as shown in Figure 7. Therefore, 100 categories are randomly selected from this branch as the first target dataset, which is denoted as DOG. Then, we randomly select 500 categories from the 549-category superclass of artifacts as a source dataset, which is denoted as far_DOG. Near_DOG is composed of the rest of the 400 (1,000-100-500) categories and 100 categories randomly selected from far_DOG. The distance between near_DOG and DOG is smaller than the distance between far_DOG and DOG, as there are many categories in near_DOG that have eyes, legs, tails, and fur (e.g., platypus, kangroo, and wolf), whereas categories in far_DOG do not have them. Next, the second target dataset is selected in a more widely 


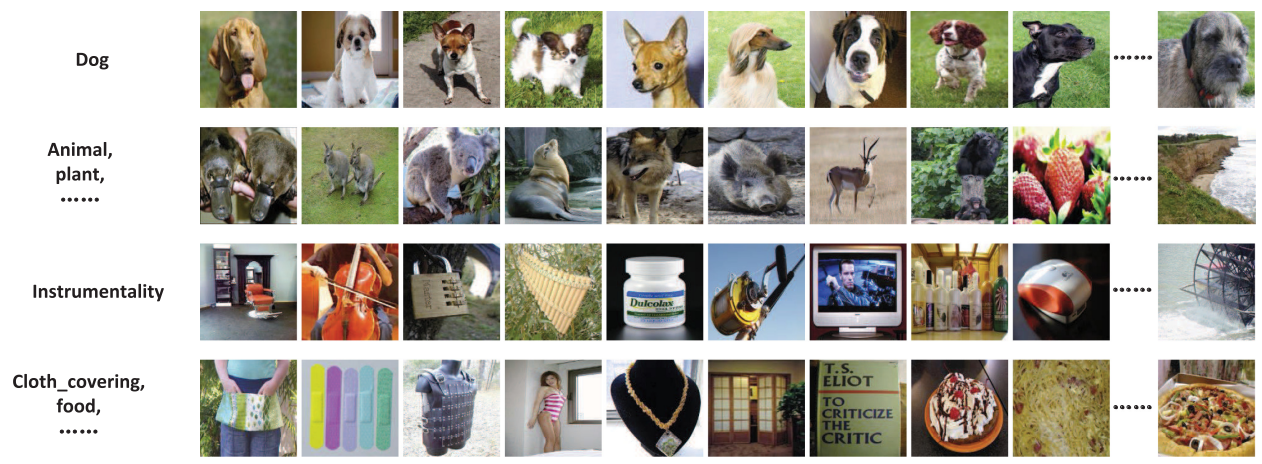

Fig. 7. Images belong to different branches. The first row shows images belonging to the superclass of dog (e.g., rhodesian ridgeback, papillon, and saint bernard). The second row shows images that are the rest of the living_thing superclass (e.g., plant, platypus, kangaroo, and wolf), except the dog class. The third row shows the superclass of instrumentality (e.g., lock, fishing pole, and mouse), and the last row shows images that belong to the rest of the artifact superclass (e.g., cloth_covering, food), except the instrumentality class.

Table 3. Performance of Fine-Tuning Different Pretrained Models on the Target Dataset (\%)

\begin{tabular}{cc|ccc}
\hline \multirow{3}{*}{ DOG } & & near_DOG & far_DOG & Scratch \\
\cline { 2 - 5 } & CNN & 59.56 & 55.94 & 55.62 \\
& SVM & $58.26(44.98)$ & $55.14(28.88)$ & 55.38 \\
\hline \multirow{3}{*}{ INSTRU } & & near_INSTRU & far_INSTRU & Scratch \\
\cline { 2 - 5 } & CNN & 67.12 & 61.58 & 54.22 \\
& SVM & $66.70(60.06)$ & $61.00(47.54)$ & 54.00 \\
\hline
\end{tabular}

Note: $\mathrm{CNN}$ indicates the accuracy that is the direct output of the CNN. SVM indicates the result of the features of layer fc7 and the combination of an SVM classifier. The performance before fine-tuning is reported in parentheses. Scratch represents the accuracy of training the model from scratch.

distributed way. As there are 347 categories in the instrumentality superclass (e.g., lock, fishing pole, and mouse), 100 categories are randomly chosen as the target dataset, which is denoted as INSTRU. The 451-categoriy superclass of living things and a random selection of 49 categories from the superclass of cloth covering and food that contain 202 categories compose far_INSTRU. Near_INSTRU contains the remaining 400 (1,000-100-500) categories and 100 categories randomly selected from far INSTRU.

The performance of fine-tuning different pretrained models on the target dataset is illustrated in Table 3. To evaluate the characteristics of different models, we also utilize the features of layer fc7 and a simple support vector machine (SVM) classifier to obtain classification accuracy on the target dataset. It can be seen that the distance between the source and target datasets has much influence on the performance of fine-tuning. For DOG recognition, the features directly extracted from the model pretrained on near_DOG $(44.98 \%)$ is much better than that of far_DOG $(28.88 \%)$. For the model that is pretrained on the source dataset that is close to the target one, it learns prior knowledge that is useful for classifying the target images. Thus, near_DOG performs better than far_DOG. Even more, the performance of fine-tuning the model that is pretrained on near_DOG (58.26) is also much better than the fine-tuned far_DOG (55.14), as expected. And the trend is the same in INSTRU. It is demonstrated that fine-tuning from a near model yields better performance 
than a far one. The results show that the more similarity there is between the source and target datasets, the better performance fine-tuning obtains.

4.3.1 Discrimination of Individual Filters. The discrimination of different layers indicates the class selectivity of the group of filters in the particular layer. Even though most of the features in CNNs are distributed code, it is also important to measure the characteristic of individual filters, as cells in the human brain have a big response to some specific and complex stimuli, which have close relationships to object recognition [3]. The discrimination of one individual filter on one class can be regarded as its sensitivity to the class. More precisely, if a filter is sensitive to one class, the filter should fire strongly on all images in this class, and at the same time it should also have a small response to images in other classes.

To measure the sensitivity of one filter for one class, we use the precision and recall curve to compute the criterion quantitatively, just like Agrawal et al. [1]. Specifically, we analyze the filters on the conv5 layer, as features of this layer are mid-level image representations, which are the combination of low-layer features and also have less semantic information compared with the features in fully connected layers. The features of conv5 consist of responses of 256 filters, which have a size of $256 \times 6 \times 6$. We use max-pooling to transform the activations of a spatial grid of size $6 \times 6$ to one value, as this strategy causes a small drop in recognition performance while bringing shorter and more compact features. With this implementation, the responses of size $256 \times 6 \times 6$ are reduced to $256 \times 1$, each element of which indicates the response of one filter. Now, for a set of images, we will get a set of associated scores. We treat every filter as a classifier and compute the precision-recall curve for each of them.

For each class, we now can get 256 curves, with each curve corresponding to one filter. The curves are computed on the test set of INSTRU, which contains 100 categories, with 50 images per class. We sort the filters in descending order of their average precision (AP values. Figure 8 shows the top-five filters of their precision-recall curves for the classes of garbage truck, forklift, and pencil box, respectively. For the garbage truck class, the top-five filters in the near model are more sensitive than the filters in far model, as the precision-recall curves of the far model are almost under the precision-recall curves of the near model. To compare one individual filter, we use the mean average precision (mAP) of the selected five filters as the sensitivity, as shown in last column of Figure 8. The mAP of near is bigger than that of far. After fine-tuning, both of the mAPs for far and near increase.

To compare these filters in different models on a global level, we calculate the number of categories (i.e., all the classes in the target dataset) that the filters in one model have bigger mAP that filters on another one to represent the difference between two models. We use sensitivity percentages to evaluate the global sensitivity of two models when comparing them. The bigger of percentage that one model has, the more sensitive the model is. Figure 9 shows the sensitivity percentages of different models on the 100 categories in INSTRU. Many conclusions can be obtained from the results.

First, filters in near are more sensitive than filters in far. As abundant visual information is put into the near model, filters in near are more sensitive than filters in self. Because the far_INSTRU dataset has big distance to the INSTRU dataset, the filters in the model trained on it (far) are more insensitive than the filters in self, and filters in far only have a bigger $\mathrm{mAP}$ in $40 \%$ categories, as shown in Figure 9(a) and (b).

Second, fine-tuning make the filters more sensitive to the target dataset. For $51 \%$ of the categories in INSTRU, the filters in the model trained on near_INSTRU (near) have a bigger mAP compared with filters in the model trained on INSTRU (self). After fine-tuning, the filters in near have a bigger mAP than filters in self in $61 \%$ of the categories, as shown in Figure 9(a). Meanwhile, Figure 9(d) 

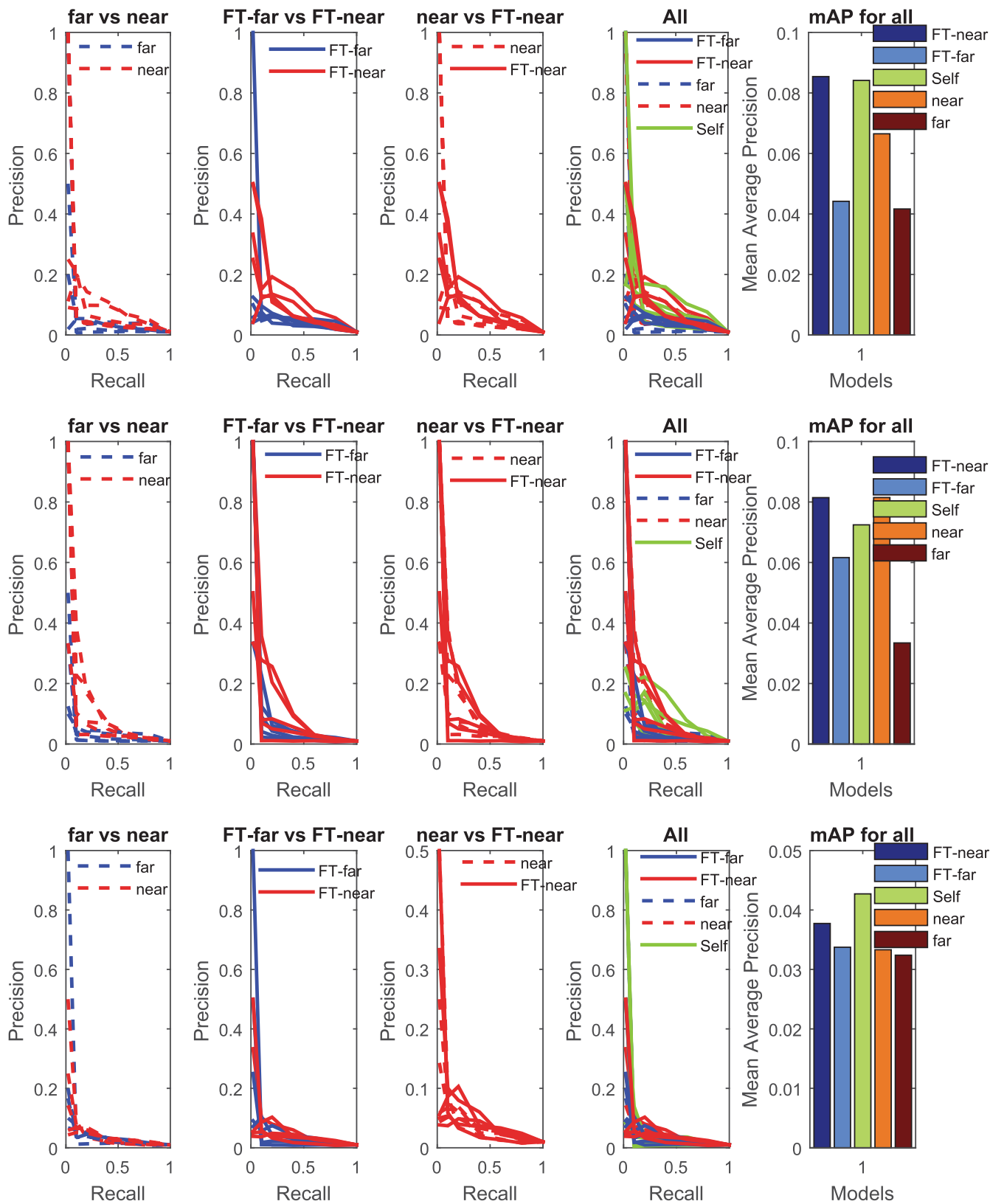

Fig. 8. The sensitivity of filters in the conv5 layer. The first row is for the class of garbage truck. The second and third rows are for the classes of forklift and pencil box, respectively. The red dashed lines in the first column shows the top-five filters in the model pretrained on near_INSTRU (near), and the blue dashed lines are for far_INSTRU (far). The red solid lines in the second column are the top-five filters after fine-tuning near on INSTRU (FT-near), and blue solid lines are for (FT-far). Red solid lines in the third column are for FT-near, and red dashed lines are for near. The fourth column shows the top-five filters in all five models. Specifically, the green solid lines are for the model trained on INSTRU itself (Self). The last column shows the mean AP of the five filters for each model. 
(a) Near vs Self

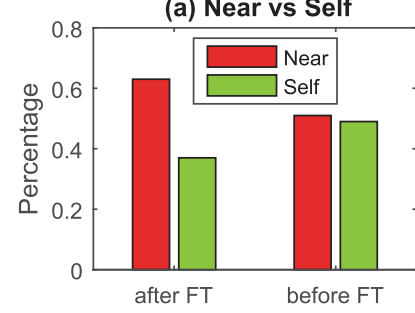

(c) Far vs Near

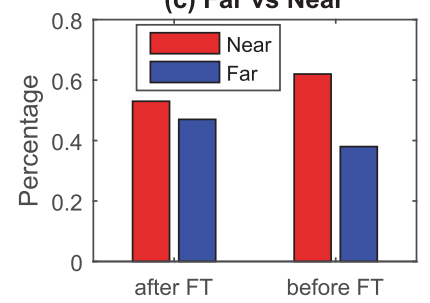

(b) Far vs Self

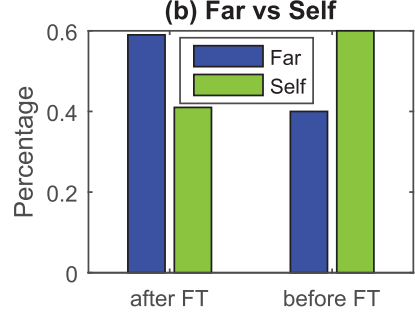

(d) After FT vs Before FT

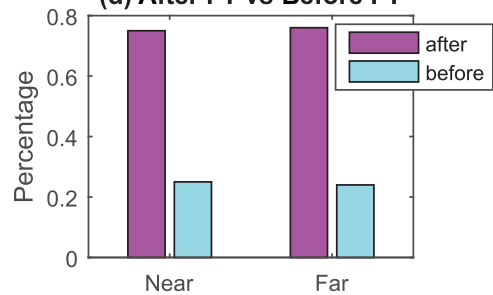

Fig. 9. The statistics of sensitivity percentages for different models on the 100 categories in INSTRU. Near represents the model first trained on near_INSTRU, and far indicates the fine model first trained on far_INSTRU. Self is the model directly trained on INSTRU from scratch.

shows that filters after fine-tuning have bigger mAP compared with filters before fine-tuning in most of the categories in both near $(75 \%)$ and far $(76 \%)$.

Last, the sensitivity difference between near and far is big, as they are optimized on different datasets focusing on different visual patterns. After fine-tuning, they both fire on the target images, so the difference between them decreases. As shown in Figure 9(c), after fine-tuning, filters in near have a big $\mathrm{mAP}$ in only $53 \%$ of the categories.

4.3.2 Discrimination of Different Layers. It is valuable to measure the discrimination of different layer features extracted from different models, as low-layer features are common for all images and high-layer features are specific to the task [60]. We analyze the classification accuracy of features extracted from layer conv1 to layer fc 8 on different models on the dataset of INSTRU. Here, conv1 represents the activation of the first convolutional layer followed by Relu, Pooling, and Norm, with a dimension of $96 \times 27 \times 27$. Conv 2 and conv5 are the same situations. We use a logistic regression classifier composed with a fully connected layer of 100 units and a softmax layer with all training examples exposed to the training procedure. The results are shown in Figure 10. Many novel conclusions can be obtained.

First, the generalization capability of the activations of a pretrained CNN model increases with the growth of the layer. In addition, if we transfer the features from a model that is trained on a source dataset that is different from the one on which we evaluate the performance (i.e., the target dataset), the activations of $\mathrm{fc} 7$ are the best. But if we train or fine-tune the model on the dataset itself, the activations of fc8 (which have smaller dimensions) are the best. As shown by the solid lines of Figure 10(a), the features of fc8 $(66.62 \%, 60.86 \%$, and 53.74 , respectively) obtain higher performance compared with the features of fc7 (66.12\%, 59.08\%, and 53.32\% respectively). However, the features of fc8 (57.78\% and $44.64 \%$, respectively) obtain lower performance in all dashed lines.

Second, for models trained on datasets that have different distances in relation to the target dataset, the performance of the near one is much higher than the performance of the far one. The case is always true for all of the layers. In addition, fine-tuning from these models, the trend remains. This corresponds with the conclusion that the more similarity there is between the source and target datasets, the better performance fine-tuning obtains. 
(a) Performance of different layers

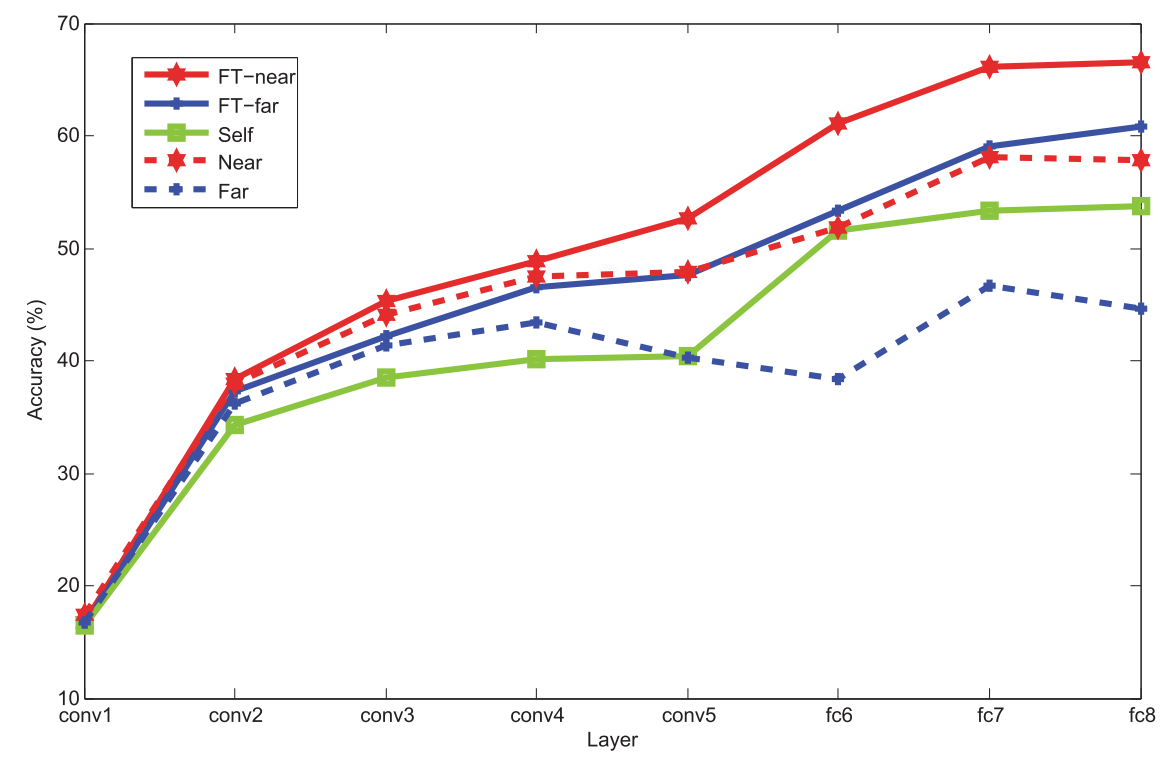

(b) The gain of the the performance
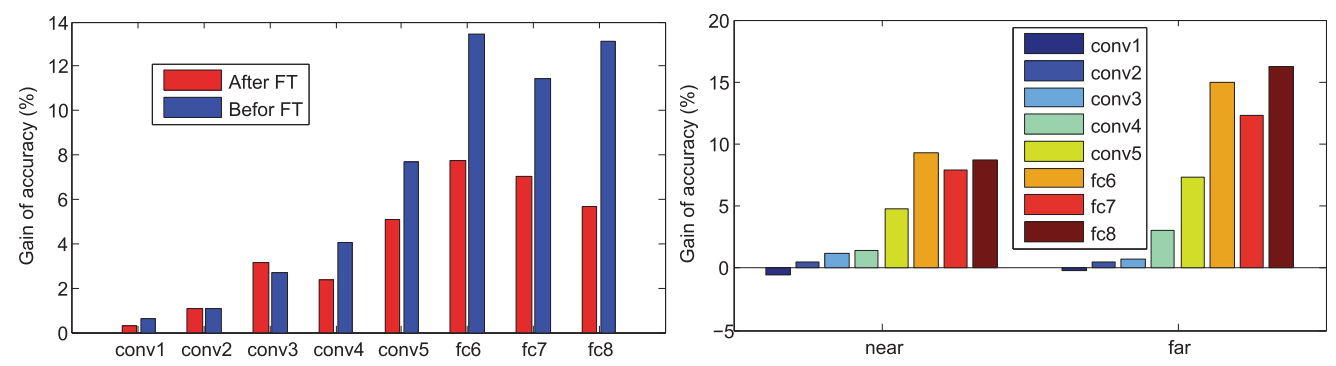

Fig. 10. The performance on INSTRU. (a) The discrimination of different layers when the features are extracted from different models. Self represents the features extracted from the model that is trained on the 100-category dataset of INSTRU. Near and far represent the model trained on the 500 categories in the dataset near_INSTRU and far_INSTRU, respectively. FT-near indicates fine-tuning the model pretrained on near_INSTRU on the target dataset, and so does FT-far. (b) The left figure illustrates the gain in performance that the near model surpasses than the far one for each layer. The right figure illustrates the gain of the performance that conducting fine-tuning (i.e., after) surpasses without fine-tuning (i.e., before) for the near and far models, respectively.

Third, the gain of the performance that the near model surpasses the far one decreases after conducting fine-tuning, as shown in the left part of Figure 10(b). The filters optimized on the dataset that is near the target dataset are sensitive to the images in the target dataset, whereas filters optimized on the far dataset seem insensitive, so the difference between these two sets of filters is big. After fine-tuning the target dataset, filters in these models both fire on the target images, so the difference decreases.

Fourth, we compare the performance of pretrained models with the one trained from scratch. Examples in the near source dataset have many common attributes or high-level visual patterns as the ones in the target dataset have. Meanwhile, the source dataset has bigger training examples $(500 \times 1,300=650,000)$ than the training examples in the target dataset $(100 \times 1,300=130,000)$, so 


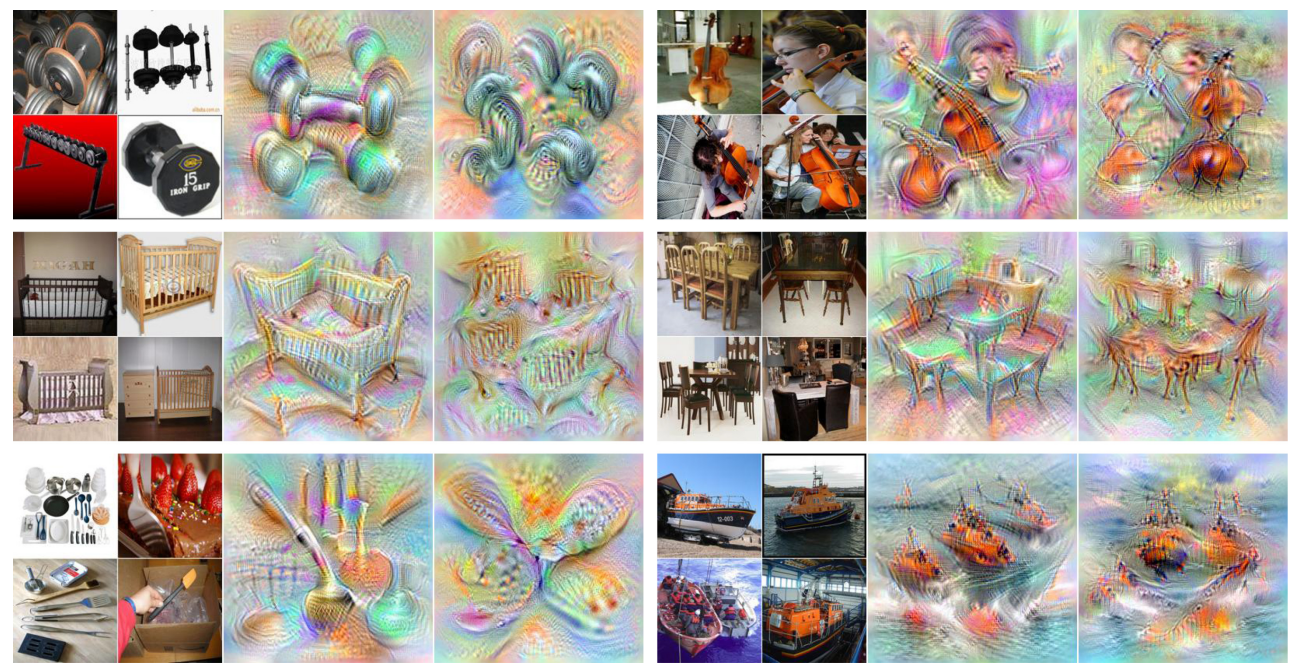

Fig. 11. The visualization of class images for different models. For each set, the first column shows same original images, and the second column is the corresponding class image computed from the model finetuned on near_INSTRU (FT-near). The third column is the corresponding image computed from the model fine-tuned on far_INSTRU (FT-far). The results show that class images computed from FT-near are better in representing the corresponding classes than the class images computed from FT-far.

representations extracted from the model trained on the source dataset (i.e., near) are better that the features extracted from the model trained on the target dataset itself (i.e., self). However, examples in the far source dataset have less common high-level visual patterns, so features extracted from the far model (Far) are inferior to the features extracted from Self. However, the performance of Far in the layers below conv5 is better than the performance of Self, as illustrated in Figure 10(a). This is because features in the low layers are common patterns for general object recognition, which have little relationship with the specific target task. With more training examples fed to Far, the model captures more simple visual patterns, leading to better performance.

4.3.3 Qualitative Analysis. We also qualitatively analyze the difference between different models. For a learned model, we utilize the method introduced by Simonyan et al. [45] to visualize the class images. More formally, let $S_{c}(I)$ be the score of the class $c$, which is computed by the classification layer of the model for image $I$. We would like to find the image $I_{c}$ such that the score $S_{c}$ is high. Thus, $I_{c}$ can be regarded as the the notion of class $c$. Figure 11 qualitatively shows some examples.

We can see that the fine-tuned near model (FT-near) learns more details of the classes in INSTRU compared with the fine-tuned far model (FT-far). For example, as shown in the first set in Figure 11, FT-far only focuses on the balls of a dumbbell. In comparison, FT-near not only focuses on the balls but also the short bar that serves as a handle. All of the examples show that the class images computed from FT-near are more representative than the ones computed from FT-far. The results demonstrate that the learned features in FT-near are better in representing images in INSTRU.

\subsection{Number of Categories and Training Examples per Category in the Target Dataset}

The number of categories and training samples per category plays an important role in training a CNN. In this section, we analyze the effect of these two factors in the target dataset when conducting fine-tuning. As the average training examples for each category in ImageNet are almost 1,200 , we fine-tune the pretrained models on target datasets with the training samples per category varying from 1,200 to 30 . At the same time, we also select 100, 80, 40,20, and 10 as the number 

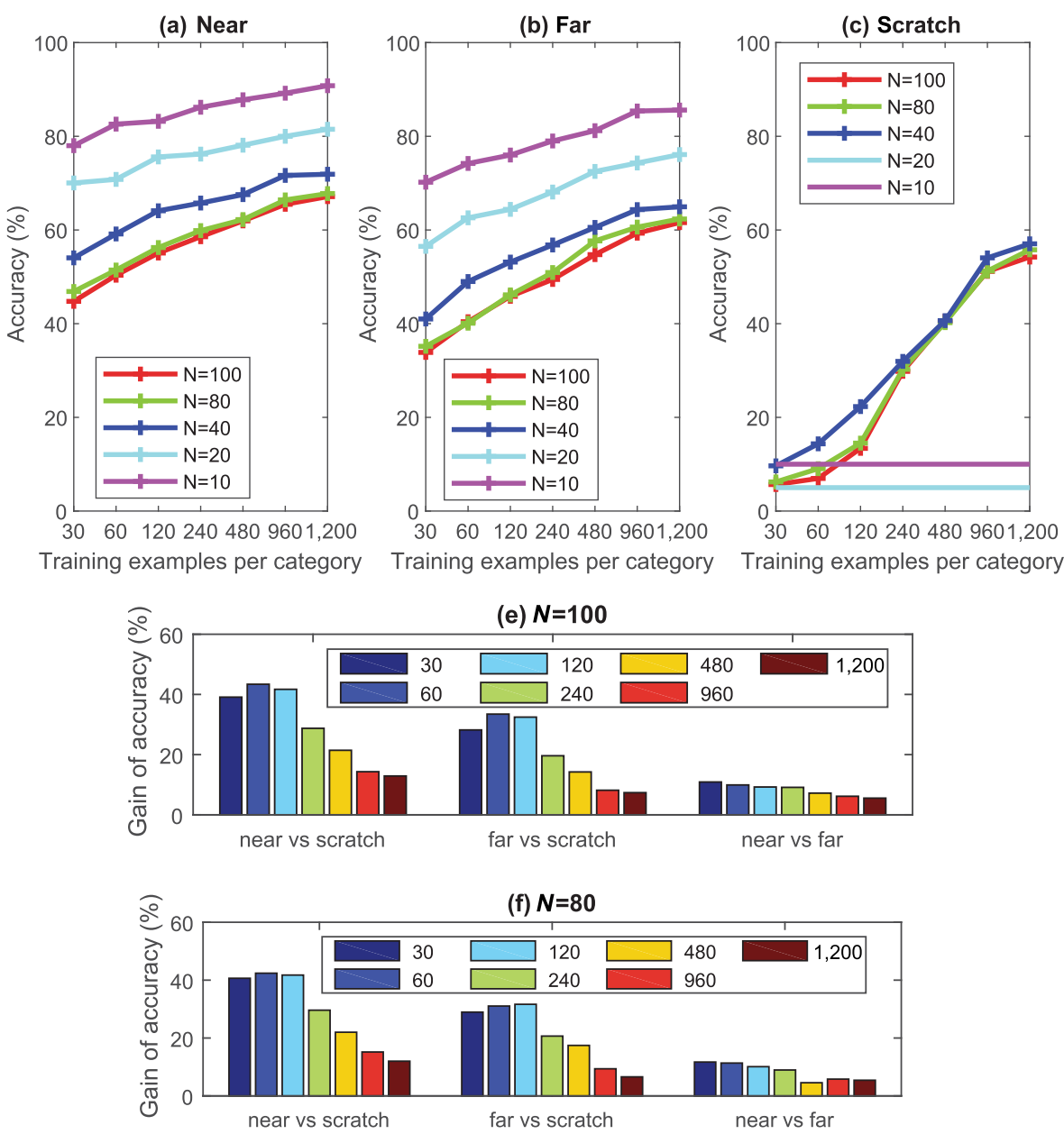

Fig. 12. The effect of the number of categories and training samples per category in the target dataset on the performance of fine-tuning. (a), (b), and (c) represent fine-tuning from near_INSTRU, fine-tuning from far_INSTRU, and training from scratch respectively. The horizontal lines in (c) represent that the models do not converge. (e) shows the comparison of two models when the number of categories in the target dataset is $100(N=100)$. For example, "near vs scratch" shows the gains of fine-tuning the model that is pretrained on near_INSTRU (near) versus training the model from scratch (scratch), with the training samples per category varying from 1,200 to 30 . (f) shows the results under the settings of $N=80$.

of categories in the target datasets, respectively. We use the models trained on far_INSTRU and near_INSTRU (introduced in Section 4.3) as two pretrained models and select target datsets with different settings from INSTRU.

As illustrated in Figure 12, the results suggest that with the decrease of the categories in the target datasets, the performance increases when conducting FT on these target datasets. This is true with both the far and near models, as well as training from scratch. With the number of categories decreasing, the accuracy may also decrease, as fewer training examples are fed to the network, which could more likely tend to overfitting. But the results illustrate that the accuracy is higher, because the classification problem is easier with fewer categories, which has fewer chances with regard to making mistakes. It is important to note that when the data are too scarce to train a 


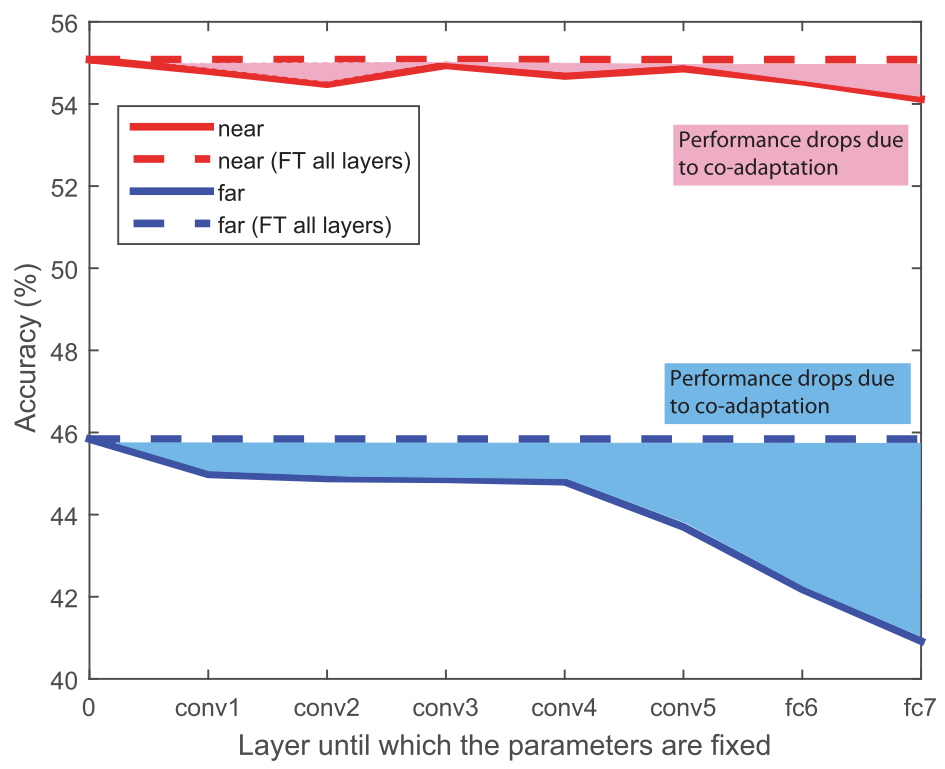

Fig. 13. The performance of fine-tuning to deep or shallow layers on different models when the training data are limited. Red and blue lines represent fine-tuning from the near model and the far model, respectively.

model, as shown in Figure 12(c), fine-tuning a pretrained model on such a small dataset can also obtain significant performance, but training from scratch does not converge. The second conclusion that we can obtain is that when the number of categories in the target dataset is fixed, the accuracy becomes higher when more training examples are exposed to the model. The results show that more training examples can provide better performance. The third conclusion is that the gain (i.e., near vs scratch, far vs scratch) decreases with the increase in training examples. The trend is the same for the gain in near versus far, as illustrated in Figure 12(e) and (f). With more training examples, the parameters in different models are both adjusted to fit the target data, being more sensitive to the images in the target dataset, so the difference decreases.

\subsection{Fine-Tuning to Deep or Shallow Layers}

Features in low layers of CNNs are general image representations such as common edges, shapes, and textures, whereas features in high layers are specific image representations for the target task. So when we fine-tune a pretrained model on the target dataset, the parameters of the model in which layer should be fixed, and which layer should be retrained? The work of Yosinski et al. [58] has suggested that fine-tuning all layers can obtain significant performance, whereas fixing the parameters of some layers will bring about the problems of co-adaptation (for middle layers) and representation specificity (for high layers). Their work provides a sufficient analysis of fine-tuning when the training examples are abundant. But what are the results when the training examples are limited? In this section, we investigate the problem of fine-tuning to deep or shallow layers in a systematic way on the condition of limited training data.

In this section, we also use the models trained on far_INSTRU and near_INSTRU (introduced in Section 4.3) as two pretrained models (denoted as near and far, respectively). For the target dataset, we use all categories in INSTRU but only sample 240 examples per category for training (the original training examples per category is about 1,200). In the experiments, the first $n$ (from 1 to 7) layers of the network are frozen. For example, when $n=3$, the parameters for the layers conv1, conv2, and conv3 are fixed during fine-tuning. The results are shown in Figure 13. The 

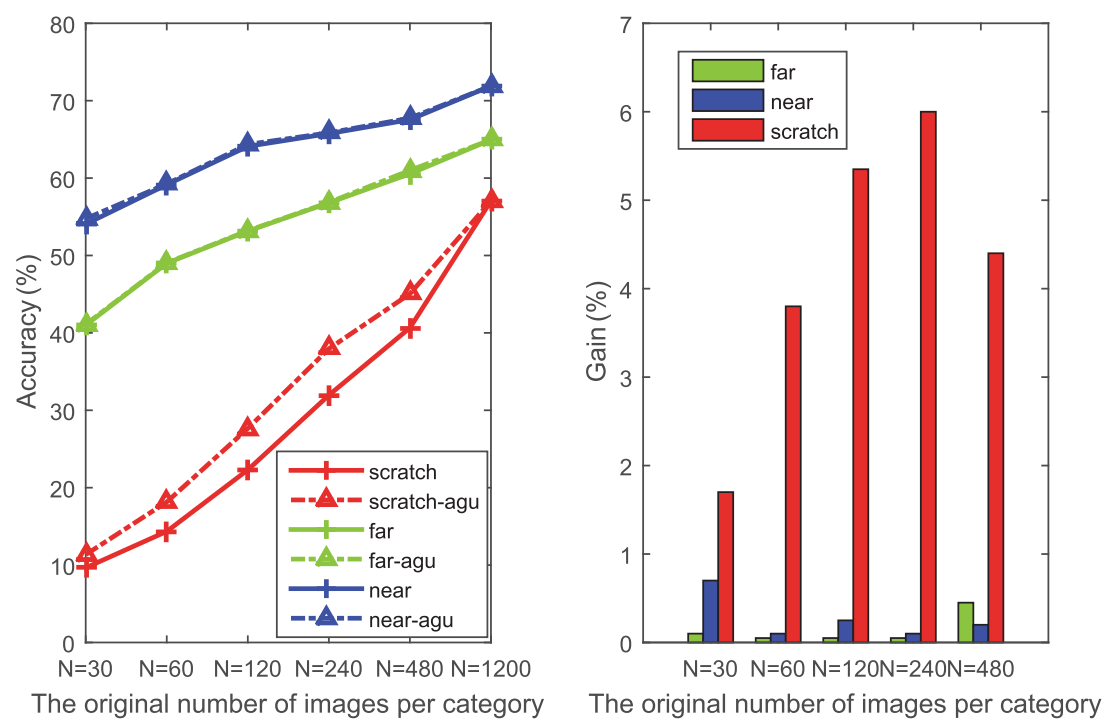

Fig. 14. The comparison of data augmentation for fine-tuning and training models from scratch. For an original number of images that is smaller than 1,200, we increase the training examples to 1,200 by data augmentation. For the left figure, red lines represent training the network from scratch. Blue and green lines represent fine-tuning the network from the near model and the far model, respectively. The right figure shows the gain that is brought by data augmentation.

first conclusion we can get is that it is better to fine-tune all layers on the target dataset. This is consistent with the conclusion in Yosinski et al. [58], where the training examples in the target dataset is large. The second conclusion is that there is less co-adaptation (i.e., features that interact with each other in a complex or fragile way such that this co-adaptation could not be relearned by the upper layers alone [58]) in the model that is fine-tuned from near compared with the model that is fine-tuned from far. This is because the parameters in near are already fitted to the target data very well. They can be easily transferred to classifying the target data. It also can be concluded that just fine-tuning the high layers such as conv5, fc6, and fc7 can yield satisfactory results when the training data is small.

\subsection{Effect of Data Augmentation}

Data augmentation is a common and simple approach to learning discriminative and representative features by augmenting the training set with transformed versions of the original images. Techniques such as cropping, rotating, scaling, and flipping original input images have been widely used for image classification. In this section, we evaluate the effect of data augmentation for finetuning. We artificially constrain our access to the target training data and evaluate the gain in recognition accuracy that is brought by data augmentation.

For the target datasets, we use all categories in INSTRU, with the original training samples per category varying from 1,200 to 30. Two pretrained models (introduced in Section 4.3, denoted as near and far) are fine-tuned on these datasets, respectively. As the original training examples per category total about 1,200 , we set 1,200 as the upper bound. In our experiments, if the original number of images per category in the training set is smaller than 1,200 , we use the data augmentation technique in Paulin et al. [40] to augment the training images to 1,200 .

The results are shown in Figure 14. We compare the gain in accuracy when training the network from scratch, fine-tuning the network from the near model and the far model, respectively. 
The conclusion is that data augmentation brings significant gains for training neural networks from scratch, whereas for fine-tuning, the gains are smaller. The performance for fine-tuning with and without data augmentation is almost the same. For training models from scratch, as models trained with small data do not generalize well, data augmentation increases the amount of training data, enhancing the ability to correctly classify images. However, a pretrained model is initialized with proper weights. Fine-tuning it on the generated examples brings small changes. Thus, data augmentation for fine-tuning is less effective than data augmentation for training networks from scratch.

\section{CONCLUSION}

Features extracted from CNNs trained on large-scale datasets are becoming common and preferred image representations for more and more visual tasks. Fine-tuning on a pretrained model not only saves lots of time to avoid training networks from scratch but also guarantees better performance. Our article is dedicated to analyzing the factors that influence the performance of fine-tuning. We constrain our access to the subsets extracted from ImageNet and explore each factor in turn. Many observations can be concluded from the results. First, with roughly the same distance to the target dataset, the bigger the number of categories in the source dataset, the better performance finetuning obtains. Second, when the source dataset is fixed, the performance of fine-tuning increases with more training examples exposed to the retraining of the pretrained model. In addition, the gain in fine-tuning versus training the network from scratch decreases with the increase in retraining examples. Third, the more similarity between the source and target datasets, the better performance fine-tuning obtains. We analyze the characteristics of different models at both filter level and layer level, and we show their sensitivities to the target dataset. These conclusions provide useful and evidence-based intuition about how to implement fine-tuning for other visual tasks. In future work, we will explore architectures with an auxiliary loss to leverage the relationship between the source and target datasets explicitly when conducting fine-tuning.

\section{REFERENCES}

[1] Pulkit Agrawal, Ross Girshick, and Jitendra Malik. 2014. Analyzing the performance of multilayer neural networks for object recognition. In Proc. Eur. Conf. Comput. Vis. 329-344.

[2] Hossein Azizpour, Ali Sharif Razavian, Josephine Sullivan, Atsuto Maki, and Stefan Carlsson. 2016. Factors of transferability for a generic convNet representation. IEEE Trans. Pattern Anal. Mach. Intell. 38, 9 (2016), 1790-1802.

[3] Horace B. Barlow. 1972. Single units and sensation: A neuron doctrine for perceptual psychology?Perception 1, 4 (1972), 371-394.

[4] Shai Ben-David, John Blitzer, Koby Crammer, Alex Kulesza, Fernando Pereira, and Jennifer Wortman Vaughan. 2010. A theory of learning from different domains. Mach. Learn. 79 (2010), 151-175.

[5] Shai Ben-David, John Blitzer, Koby Crammer, and Fernando Pereira. 2007. Analysis of representations for domain adaptation. In Proc. Adv. Neural Inf. Process. Syst. 137-144.

[6] Yoshua Bengio, Pascal Lamblin, Dan Popovici, and Hugo Larochelle. 2007. Greedy layer-wise training of deep networks. In Proc. Adv. Neural Inf. Process. Syst. 153-160.

[7] Ken Chatfield, Karen Simonyan, Andrea Vedaldi, and Andrew Zisserman. 2014. Return of the devil in the details: Delving deep into convolutional nets. In Proc. Brit. Mach. Vis. Conf.

[8] Sumit Chopra, Suhrid Balakrishnan, and Raghuraman Gopalan. 2013. DLID: Deep learning for domain adaptation by interpolating between domains. In Proc. 30th Int. Conf. Mach. Learn. Workshop Represent. Learn., Vol. 2.

[9] Jeff Donahue, Yangqing Jia, Oriol Vinyals, Judy Hoffman, Ning Zhang, Eric Tzeng, and Trevor Darrell. 2014. DeCAF: A deep convolutional activation feature for generic visual recognition. In Proc. 31th Int. Conf. Mach. Learn. 647-655.

[10] Dumitru Erhan, Yoshua Bengio, Aaron Courville, Pierre-Antoine Manzagol, Pascal Vincent, and Samy Bengio. 2010. Why does unsupervised pre-training help deep learning? Jour. Mach. Learn. Research 11 (2010), 625-660.

[11] Ross Girshick. 2015. Fast R-CNN. In Proc. IEEE Int. Conf. Comput. Vis. 1440-1448.

[12] Ross Girshick, Jeff Donahue, Trevor Darrell, and Jitendra Malik. 2014. Rich feature hierarchies for accurate object detection and semantic segmentation. In Proc. IEEE Conf. Comput. Vis. Pattern Recognit. 580-587. 
[13] Xavier Glorot, Antoine Bordes, and Yoshua Bengio. 2011. Domain adaptation for large-scale sentiment classification: A deep learning approach. In Proc. 28th Int. Conf. Mach. Learn. 513-520.

[14] Yunchao Gong, Liwei Wang, Ruiqi Guo, and Svetlana Lazebnik. 2014. Multi-scale orderless pooling of deep convolutional activation features. In Proc. Eur. Conf. Comput. Vis. 392-407.

[15] Saurabh Gupta, Ross Girshick, Pablo Arbeláez, and Jitendra Malik. 2014. Learning rich features from RGB-D images for object detection and segmentation. In Proc. Eur. Conf. Comput. Vis. 345-360.

[16] Steven Gutstein, Olac Fuentes, and Eric Freudenthal. 2008. Knowledge transfer in deep convolutional neural net. Int. four. Artif. Intel. Tool. 17, 3 (2008), 555-567.

[17] Kaiming He, Xiangyu Zhang, Shaoqing Ren, and Jian Sun. 2014. Spatial pyramid pooling in deep convolutional networks for visual recognition. In Proc. Eur. Conf. Comput. Vis. 346-361.

[18] Kaiming He, Xiangyu Zhang, Shaoqing Ren, and Jian Sun. 2016. Deep residual learning for image recognition. In Proc. IEEE Conf. Comput. Vis. Pattern Recognit. 770-778.

[19] Kaiming He, Xiangyu Zhang, Shaoqing Ren, and Jian Sun. 2016. Identity mappings in deep residual networks. In Proc. Eur. Conf. Comput. Vis. 630-645.

[20] Geoffrey E. Hinton and Ruslan R. Salakhutdinov. 2006. Reducing the dimensionality of data with neural networks. Science 313, 5786 (2006), 504-507.

[21] Seunghoon Hong, Junhyuk Oh, Honglak Lee, and Bohyung Han. 2016. Learning transferrable knowledge for semantic segmentation with deep convolutional neural network. In Proc. IEEE Conf. Comput. Vis. Pattern Recognit. 3204-3212.

[22] Jie Hu, Li Shen, and Gang Sun. 2018. Squeeze-and-excitation networks. In Proc. IEEE Conf. Comput. Vis. Pattern Recognit. 7132-7141.

[23] Gao Huang, Zhuang Liu, Kilian Q. Weinberger, and Laurens van der Maaten. 2017. Densely connected convolutional networks. In Proc. IEEE Conf. Comput. Vis. Pattern Recognit. 4700-4708.

[24] Sergey Ioffe and Christian Szegedy. 2015. Batch normalization: Accelerating deep network training by reducing internal covariate shift. In Proc. 32th Int. Conf. Mach. Learn. 448-456.

[25] Yangqing Jia, Evan Shelhamer, Jeff Donahue, Sergey Karayev, Jonathan Long, Ross Girshick, Sergio Guadarrama, and Trevor Darrell. 2014. Caffe: Convolutional architecture for fast feature embedding. In Proc. 22nd ACM Int. Conf. Multimedia. 675-678.

[26] Alex Krizhevsky, Ilya Sutskever, and Geoffrey E. Hinton. 2012. ImageNet classification with deep convolutional neural networks. In Proc. Adv. Neural Inf. Process. Syst. 1097-1105.

[27] Xiangyang Li, Shuqiang Jiang, Xinhang Song, Luis Herranz, and Zhiping Shi. 2014. Multipath convolutional-recursive neural networks for object recognition. In Proc. Int. Conf. Intelligent Information Processing, Vol. 432. 269-277.

[28] Min Lin, Qiang Chen, and Shuichen Yan. 2014. Network in network. In Proc. Int. Conf. Learn. Representations.

[29] Heng Liu, Zilin Fu, Jungong Han, Ling Shao, Shudong Hou, and Yuezhong Chu. 2019. Single image super-resolution using multi-scale deep encoder-decoder with phase congruency edge map guidance. Inf. Sci. 473 (2019), 44-58.

[30] Lingqiao Liu, Chunhua Shen, and Anton van den Hengel. 2015. The treasure beneath convolutional layers: Crossconvolutional-layer pooling for image classification. In Proc. IEEE Conf. Comput. Vis. Pattern Recognit. 4749-4757.

[31] Jonathan Long, Evan Shelhamer, and Trevor Darrell. 2015. Fully convolutional networks for semantic segmentation. In Proc. IEEE Conf. Comput. Vis. Pattern Recognit. 3431-3440.

[32] Mingsheng Long, Yue Cao, Jianmin Wang, and Michael Jordan. 2015. Learning transferable features with deep adaptation networks. In Proc. 32th Int. Conf. Mach. Learn. 97-105.

[33] Shangzhen Luan, Chen Chen, Baochang Zhang, Jungong Han, and Jianzhuang Liu. 2018. Gabor convolutional networks. IEEE Trans. Imag. Proces. 27, 9 (2018), 4357-4366.

[34] George A. Miller. 1995. WordNet: A lexical database for English. Commun. ACM 38, 11 (1995), 39-41.

[35] Maxime Oquab, Leon Bottou, Ivan Laptev, and Josef Sivic. 2014. Learning and transferring mid-level image representations using convolutional neural networks. In Proc. IEEE Conf. Comput. Vis. Pattern Recognit. 1717-1724.

[36] Wanli Ouyang, Xiaogang Wang, Cong Zhang, and Xiaokang Yang. 2016. Factors in finetuning deep model for object detection with long-tail distribution. In Proc. IEEE Conf. Comput. Vis. Pattern Recognit. 864-873.

[37] Sinno Jialin Pan and Qiang Yang. 2010. A survey on transfer learning. IEE Trans. Knowl. Data Eng. 22, 10 (2010), 1345-1359.

[38] Vishal M. Patel, Raghuraman Gopalan, Ruonan Li, and Rama Chellappa. 2015. Visual domain adaptation: A survey of recent advances. IEEE Signal Process. Mag. 32, 3 (2015), 53-69.

[39] Novi Patricia and Barbara Caputo. 2014. Learning to learn, from transfer learning to domain adaptation: A unifying perspective. In Proc. IEEE Conf. Comput. Vis. Pattern Recognit. 1442-1449.

[40] Mattis Paulin, Jérôme Revaud, Zaid Harchaoui, Florent Perronnin, and Cordelia Schmid. 2014. Transformation pursuit for image classification. In Proc. IEEE Conf. Comput. Vis. Pattern Recognit. 3646-3653.

[41] Shaoqing Ren, Kaiming He, Ross Girshick, and Jian Sun. 2015. Faster R-CNN: Towards real-time object detection with region proposal networks. In Proc. Adv. Neural Inf. Process. Syst. 91-99. 
[42] Olga Russakovsky, Jia Deng, Hao Su, Jonathan Krause, Sanjeev Satheesh, Sean Ma, Zhiheng Huang, et al. 2015. ImageNet large scale visual recognition challenge. Int. four. Comp. Vis. 115, 3 (2015), 211-252.

[43] Pierre Sermanet, David Eigen, Xiang Zhang, Michael Mathieu, Rob Fergus, and Yann LeCun. 2014. Overfeat: Integrated recognition, localization and detection using convolutional networks. In Proc. Int. Conf. Learn. Representations.

[44] Ali Sharif Razavian, Hossein Azizpour, Josephine Sullivan, and Stefan Carlsson. 2014. CNN features off-the-shelf: An astounding baseline for recognition. In Proc. IEEE Conf. Comput. Vis. Pattern Recognit. Workshop. 806-813.

[45] Karen Simonyan, Andrea Vedaldi, and Andrew Zisserman. 2014. Deep inside convolutional networks: Visualising image classification models and saliency maps. In Proc. Int. Conf. Learn. Representations Workshop.

[46] Karen Simonyan and Andrew Zisserman. 2015. Very deep convolutional networks for large-scale image recognition. In Proc. Int. Conf. Learn. Representations.

[47] Christian Szegedy, Sergey Ioffe, Vincent Vanhoucke, and Alexander A. Alemi. 2017. Inception-v4, inception-ResNet and the impact of residual connections on learning. In Proc. 31st AAAI Conf. Arti. Intellig. 4278-4284.

[48] Christian Szegedy, Wei Liu, Yangqing Jia, Pierre Sermanet, Scott Reed, Dragomir Anguelov, Dumitru Erhan, Vincent Vanhoucke, and Andrew Rabinovich. 2015. Going deeper with convolutions. In Proc. IEEE Conf. Comput. Vis. Pattern Recognit. 1-9.

[49] Antonio Torralba and Alexei A. Efros. 2011. Unbiased look at dataset bias. In Proc. IEEE Conf. Comput. Vis. Pattern Recognit. 1521-1528.

[50] Eric Tzeng, Judy Hoffman, Ning Zhang, Kate Saenko, and Trevor Darrell. 2014. Deep domain confusion: Maximizing for domain invariance. arXiv:1412.3474. http://arxiv.org/abs/1412.3474

[51] Pascal Vincent, Hugo Larochelle, Yoshua Bengio, and Pierre-Antoine Manzagol. 2008. Extracting and composing robust features with denoising autoencoders. In Proc. 25th Int. Conf. Mach. Learn. 1096-1103.

[52] Raimar Wagner, Markus Thom, Roland Schweiger, Gunther Palm, and Albrecht Rothermel. 2013. Learning convolutional neural networks from few samples. In Proc. Int. Joint Conf. Neural Netw. 1-7.

[53] Yu-Xiong Wang, Deva Ramanan, and Martial Hebert. 2017. Growing a brain: Fine-tuning by increasing model capacity. In Proc. IEEE Conf. Comput. Vis. Pattern Recognit. 2471-2480.

[54] Gengshen Wu, Jungong Han, Yuchen Guo, Li Liu, Guiguang Ding, Qiang Ni, and Ling Shao. 2019. Unsupervised deep video hashing via balanced code for large-scale video retrieval. IEEE Tran. Imag. Proces. 28, 4 (2019), 1993-2007.

[55] Gengshen Wu, Jungong Han, Zijia Lin, Guiguang Ding, Baochang Zhang, and Qiang Ni. 2018. Joint image-text hashing for fast large-scale cross-media retrieval using self-supervised deep learning. IEEE Trans. Indust. Elect. 66, 2 (2018), 9868-9877. DOI : https://doi.org/10.1109/TIE.2018.2873547

[56] Gengshen Wu, Zijia Lin, Jungong Han, Li Liu, Guiguang Ding, Baochang Zhang, and jialie Shen. 2018. Unsupervised deep hashing via binary latent factor models for large-scale cross-modal retrieval. In Proc. 27th Inter. Foint Conf. Artif. Intel. 2854-2860.

[57] Donggeun Yoo, Sunggyun Park, Joon-Young Lee, and In So Kweon. 2015. Multi-scale pyramid pooling for deep convolutional representation. In Proc. IEEE Conf. Comput. Vis. Pattern Recognit. Workshop. 71-80.

[58] Jason Yosinski, Jeff Clune, Yoshua Bengio, and Hod Lipson. 2014. How transferable are features in deep neural networks? In Proc. Adv. Neural Inf. Process. Syst. 3320-3328.

[59] Amir R. Zamir, Alexander Sax, William Shen, Leonidas Guibas, Jitendra Malik, and Silvio Savarese. 2018. Taskonomy: Disentangling task transfer learning. In Proc. IEEE Conf. Comput. Vis. Pattern Recognit. 3712-3722.

[60] Matthew D. Zeiler and Rob Fergus. 2014. Visualizing and understanding convolutional networks. In Proc. Eur. Conf. Comput. Vis. 818-833.

[61] Bolei Zhou, Agata Lapedriza, Aditya Khosla, Aude Oliva, and Antonio Torralba. 2017. Places: A 10 million image database for scene recognition. IEEE Trans. Pattern Anal. Mach. Intell. 40, 6 (2017), 1452-1464.

[62] Zongwei Zhou, Jae Shin, Lei Zhang, Suryakanth Gurudu, Michael Gotway, and Jianming Liang. 2017. Fine-tuning convolutional neural networks for biomedical image analysis: Actively and incrementally. In Proc. IEEE Conf. Comput. Vis. Pattern Recognit. 7340-7351.

Received July 2018; revised December 2018; accepted March 2019 